\title{
VELOCITY-BASED GAIN-SCHEDULED LATERAL AUTO-PILOT FOR AN AGILE MISSILE
}

\author{
D.J.Leith ${ }^{*}$, A.Tsourdos ${ }^{\dagger}$, B.A.White ${ }^{\dagger}$ W.E.Leithead ${ }^{*}$ \\ *Dept. of Electronic \& Electrical Engineering, University of Strathclyde, U.K. \\ ${ }^{\dagger}$ Dept. of Aerospace, Power \& Sensors, Cranfield University-RMCS, U.K.
}

\begin{abstract}
This paper investigates the application of velocity-based gain-scheduling techniques to a demanding, highly nonlinear, missile control design task. Scheduling on instantaneous incidence (a rapidly varying quantity) is well known to lead to considerable difficulties with conventional gain-scheduling methods. Moreover, the missile dynamics do not have well-defined relative degree at operating points where the lateral velocity is zero and thus conventional feedback linearisation methods cannot be applied to design a controller.
\end{abstract}

\section{INTRODUCTION}

Gain-scheduling control is widely employed in flight control applications, where high performance has to be achieved over a broad operating envelope. In the classical gain-scheduling design approach, a non-linear controller is constructed by continuously interpolating, in some manner, between the members of a family of linear controllers. Each linear controller is, typically, associated with a specific equilibrium operating point of the missile and is designed to ensure that, locally to the equilibrium operating point, the performance requirements are met. By employing a series expansion linearisation which, locally to the equilibrium operating point, has similar dynamics to the aircraft, linear techniques may be used to resolve this local design task. Continuity is, therefore, maintained with established linear design techniques for which a considerable body of experience has been accumulated. While this traditional gain-scheduling approach is extremely successful in most flight control applications (McLean 1990), the trend is towards vehicle configurations where the conventional gain-scheduling conditions may not always be satisfied. Gain-scheduled controllers are traditionally designed on the basis of the dynamics relative to a family of trim conditions assuming that the airspeed is slowly varying. However, during aggressive manoeuvring the vehicle may be far from equilibrium with rapidly varying airspeed. In addition, the requirement to operate at high angles of attack can necessitate scheduling on rapidly varying quantities such as the instantaneous incidence angle. It should be noted that scheduling on instantaneous incidence is well known to be problematical and is almost always avoided in classical scheduling arrangements. Specifically, in the example considered in this paper, a conventional gain-scheduling design approach fails to lead to a stabilising controller.

Faced with these kind of issues (which are also relevant in many other applications), an recent years a number of alternative approaches have been proposed which attempt to extend gain-scheduling methods including those based on local model networks and Takagi-Sugeno fuzzy models (see, for example, Johansen \& Murray-Smith 1997, Hunt \& Johansen 
1997). However, the latter typically include offequilibrium information at the cost of moving to nonlinear (especially affine) formulations and so lose the continuity with linear methods which is one of the principle advantages of classical gain-scheduling. Moreover, the requirement for some form of slow variation condition is often retained either directly or indirectly via assumptions implicit in the formulation (Leith \& Leithead 1999a). The velocity-based analysis and design framework, recently proposed in Leith \& Leithead (1998a,b), associates a linear system with every operating point of a nonlinear system, not just the equilibrium operating points. This approach thereby relaxes the restriction to near equilibrium operation while maintaining the continuity with linear methods as required. Moreover, it can be shown that the velocitybased approach does not inherently involve any slow variation requirement (Leith \& Leithead 1999b). Velocity-based techniques provide a useful bridge between classical control approaches and AI methods such as those based on local model networks and Takagi-Sugeno fuzzy models, generalising the series expansion linearisation which forms the basis of the former while retaining the blended multiple model structure of the latter. The aim of this paper is to investigate the application of velocity-based gainscheduling techniques to a demanding, highly nonlinear, missile control design task.

\section{MODELLING FOR CONTROL}

The missile lateral dynamics are (White et al. 1998, Tsourdos et al. 1998)

$$
\begin{aligned}
& \dot{\mathrm{r}}=\left(\mathrm{b}_{4}|\mathrm{v}|+\mathrm{b}_{5}\right) \mathrm{r}+\left(\mathrm{b}_{1} \mathrm{v}^{2}+\mathrm{b}_{2}|\mathrm{v}|+\mathrm{b}_{3}\right) \mathrm{v}+\left(\mathrm{b}_{6}|\mathrm{v}|+\mathrm{b}_{7}\right) \mathrm{u} \\
& \dot{\mathrm{v}}=\mathrm{a}_{3} \mathrm{r}+\left(\mathrm{a}_{1}+\mathrm{a}_{2}|\mathrm{v}|\right) \mathrm{v}+\left(\mathrm{a}_{4}|\mathrm{v}|+\mathrm{a}_{5}\right) \mathrm{u} \\
& \eta_{\mathrm{y}}=\dot{\mathrm{v}}+\mathrm{U}_{\mathrm{o}} \mathrm{r}=\left(\mathrm{a}_{1}+\mathrm{a}_{2}|\mathrm{v}|\right) \mathrm{v}+\left(\mathrm{a}_{4}|\mathrm{v}|+\mathrm{a}_{5}\right) \mathrm{u}
\end{aligned}
$$

where $r$ is the yaw rate $(\mathrm{rad} / \mathrm{s})$, $v$ the lateral velocity $(\mathrm{m} / \mathrm{s}), \eta_{\mathrm{y}}$ the lateral acceleration $\left(\mathrm{m} / \mathrm{s}^{2}\right)$, $u$ the fin angle ( $\mathrm{rad}$ ) and $\mathrm{U}_{\mathrm{o}}$ is the nominal forward speed $(\mathrm{m} / \mathrm{s})$. (Note that the missile dynamics do not have well-defined relative degree at operating points where the lateral velocity is zero and thus conventional feedback linearisation methods cannot be applied to design a controller). Differentiating, the corresponding velocitybased formulation is obtained

$$
\begin{gathered}
\dot{\mathrm{w}}_{\mathrm{r}}=\left(\mathrm{b}_{4}|\mathrm{v}|+\mathrm{b}_{5}\right) \mathrm{w}_{\mathrm{r}}+\left(3 \mathrm{~b}_{1} \mathrm{v}^{2}+2 \mathrm{~b}_{2}|\mathrm{v}|+\mathrm{b}_{3}+\mathrm{b}_{4} \mathrm{rggn}(\mathrm{v})+\mathrm{b}_{6} \mathrm{u} \operatorname{sgn}(\mathrm{v})\right) \mathrm{w}_{\mathrm{v}} \\
+\left(\mathrm{b}_{6}|\mathrm{v}|+\mathrm{b}_{7}\right) \dot{\mathrm{u}} \\
\dot{\mathrm{w}}_{\mathrm{v}}=\mathrm{a}_{3} \mathrm{w}_{\mathrm{r}}+\left(\mathrm{a}_{1}+2 \mathrm{a}_{2}|\mathrm{v}|+\mathrm{a}_{4} \mathrm{u} \operatorname{sgn}(\mathrm{v})\right) \mathrm{w}_{\mathrm{v}}+\left(\mathrm{a}_{4}|\mathrm{v}|+\mathrm{a}_{5}\right) \dot{\mathrm{u}} \\
\dot{\eta}_{\mathrm{y}}=\left(\mathrm{a}_{1}+2 \mathrm{a}_{2}|\mathrm{v}|+\mathrm{a}_{4} \mathrm{u} \operatorname{sgn}(\mathrm{v})\right) \mathrm{w}_{\mathrm{v}}+\left(\mathrm{a}_{4}|\mathrm{v}|+\mathrm{a}_{5}\right) \dot{\mathrm{u}}
\end{gathered}
$$

with $\dot{r}=w_{r}, \dot{v}=w_{v}$. The velocity-based linearisation associated with an operating point is obtained by simply "freezing" (2) at the relevant operating point and the collection of such linearisations forms the velocitybased linearisation family. The solution to the velocity-based linearisation associated with a particular operating point locally approximates the solution to the nonlinear dynamics, (1), and the solutions to the velocity-based linearisation family can be pieced together to recover the global solution to (1). The velocity-based framework therefore allows continuity with established linear methods to be maintained even in the nonlinear context.

Since there are a continuum of operating points, the velocity-based linearisation family associated with a nonlinear system has, of course, infinitely many members. It is therefore attractive to determine a (perhaps approximate) finite parameterisation of the family on which to base control designs. This requirement leads naturally to consideration of a blended multiple model representation of the linearisation family whereby the linearisations at a small number of representative operating points are blended together/interpolated between to produce an approximation to the exact linearisation family. Such a representation is closely related to neuro-fuzzy modelling approaches including Local Model Networks and Takagi-Sugeno fuzzy methods . However, in contrast to the latter methods, the velocity-based representation (Leith \& Leithead 1999a)

- Uses genuinely linear local models (not affine).

- The dynamics are directly related to the local models: the solution to a velocity-based blended multiple model system, locally to a specific operating point, is described by the solution to the linear system obtained by "freezing" the blended multiple model system at the relevant operating point. The resulting frozen system is simply a weighted linear combination of the local models.

- A still stronger property is that the solution to the blended multiple model system, locally to a specific operating point, is approximated by the weighted linear combination of the solutions to the local models.

In the present application, the velocity-based linearisation family associated with the missile lateral dynamics is approximated by the blended family

$$
\left[\begin{array}{c}
\dot{\hat{\mathrm{w}}}_{\mathrm{r}} \\
\dot{\hat{\mathrm{w}}}_{\mathrm{v}}
\end{array}\right]=\mathbf{A}(\mathrm{v})\left[\begin{array}{c}
\hat{\mathrm{w}}_{\mathrm{r}} \\
\hat{\mathrm{w}}_{\mathrm{v}}
\end{array}\right]+\mathbf{B}(\mathrm{v}) \dot{\mathrm{u}}
$$

with $\mathbf{A}(\mathrm{v})=\sum_{\mathrm{i}=1}^{5} \mathrm{w}_{\mathrm{i}}(|\mathrm{v}|) \mathbf{A}_{\mathrm{i}}, \mathbf{B}(\mathrm{v})=\sum_{\mathrm{i}=1}^{5} \mathrm{w}_{\mathrm{i}}(|\mathrm{v}|) \mathbf{B}_{\mathrm{i}}$

$$
\begin{aligned}
& \text { where } \mathbf{A}_{1}=\left[\begin{array}{cc}
-3.38 & 0.62 \\
-1000 & -3.24
\end{array}\right], \mathbf{A}_{2}=\left[\begin{array}{cc}
-2.85 & 5.70 \\
-1000 & -11.88
\end{array}\right] \\
& \mathbf{A}_{3}=\left[\begin{array}{cc}
-2.32 & 15.37 \\
-1000 & -20.52
\end{array}\right], \mathbf{A}_{4}=\left[\begin{array}{cc}
-1.25 & 48.47 \\
-1000 & -37.79
\end{array}\right], \mathbf{A}_{5}=\left[\begin{array}{cc}
-0.18 & 99.92 \\
-1000 & -55.07
\end{array}\right] \\
& \mathbf{B}_{1}=\left[\begin{array}{c}
5502.8 \\
-2830.0
\end{array}\right], \mathbf{B}_{2}=\left[\begin{array}{c}
5166.8 \\
-2657.2
\end{array}\right] \\
& \mathbf{B}_{3}=\left[\begin{array}{c}
4830.8 \\
-2484.4
\end{array}\right], \mathbf{B}_{4}=\left[\begin{array}{c}
4158.8 \\
-2138.8
\end{array}\right], \mathbf{B}_{5}=\left[\begin{array}{c}
3486.8 \\
-1793.2
\end{array}\right]
\end{aligned}
$$


The coefficients in (2) which depend on yaw rate, $r$, and fin angle, $\mathrm{u}$, are neglected so that the coefficients in (3) vary only with lateral velocity. For simplicity, standard triangular membership functions, $\mathrm{w}_{\mathrm{i}}$, centred on lateral velocities of $0 \mathrm{~m} / \mathrm{s}, 10 \mathrm{~m} / \mathrm{s}, 20 \mathrm{~m} / \mathrm{s}, 40 \mathrm{~m} / \mathrm{s}$ and $60 \mathrm{~m} / \mathrm{s}$ are used with overlap occurring only between the weighting functions associated with neighbouring centres. This scheme corresponds to straightforward linear interpolation between the local models. Despite the very strongly nonlinear nature of the dynamics, only five local models are sufficient to capture the dynamics through the entire operating envelope up to around $\pm 60 \mathrm{~g}$ (the accuracy of the approximation can be assessed by comparing the transfer functions of the linearisations associated with (2) and (3) over a range of operating points but plots are omitted here owing to space considerations). Note that provided (3) is a sufficiently accurate approximation to the exact family and/or the controller is sufficiently robust, a controller which achieves the performance objectives with these approximate dynamics is also guaranteed to achieve satisfactory performance when used with the exact plant dynamics, (1).

\section{VELOCITY-BASED CONTROL DESIGN}

The requirement is to achieve a closed-loop acceleration response with rise time of around 0.1 seconds with less that $20 \%$ overshoot. This requirement is to be satisfied over the full of operating envelope of $\pm 50 \mathrm{~g}$. The control design considered adopts an inner/outer loop type of structure common in flight control applications. A lateral velocity inner loop is designed first and then enclosed within an acceleration outer loop. The inner loop is designed to have sufficiently higher bandwidth than the outer loop that the design of the two loops can be effectively decoupled. One immediate advantage of this approach is that the dynamics from fin angle to lateral velocity are minimum-phase (the dynamics from fin to lateral acceleration are non-minimum-phase) and so an inverse can be determined directly without the need to use a minimum-phase approximation.

\section{Inner-loop}

The structure of the inner controller is shown in Fig. 1. Design of $\hat{\mathrm{G}}^{-1}$ and $\hat{\mathrm{A}}^{-1}$ The linear dynamics, $\hat{\mathrm{A}}^{-1}$, are an approximate pole-zero inverse of the actuator dynamics while $\hat{\mathrm{G}}^{-1}$ is approximately inverts the nonlinear dynamics associated with velocity-based linearisation family, (3). $\quad \hat{\mathrm{G}}^{-1}$ is designed using the velocity-based inversion approach of Leith \& Leithead (1999b). Velocity-based linearisation families are associated with the plant and the controller. Consider selecting the controller velocity-based linearisations such that at every operating point the closed-loop combination of the plant and controller linearisations has the approximately the same transfer function. The closed-loop linearisation family then has similar transfer function at every operating point. This is, however, not sufficient to ensure that the controller approximately inverts the plant dynamics. The transfer function only specifies the realisation of a linearisation to within a linear state transformation. It is, therefore, also necessary to ensure that the state-space realisations of the members of the controller linearisation family are suitably compatible with one another. (When the rate of variation of a nonlinear system is sufficiently slow, it can be shown that the controller becomes insensitive to the choice of realisation. However, in the present example the plant dynamics are rapidly varying and the controller dynamics are highly sensitive to the choice of realisation. Indeed, it is not difficult to find choices of realisation which lead to controllers that fail to an unstable closed-loop). In the present example, an appropriate realisation of approximate velocity-based inverse is

$$
\begin{aligned}
& \dot{\mathbf{w}}^{\mathrm{i}}=\mathbf{A}^{\mathrm{i}}(\mathrm{v}) \mathbf{w}+\mathbf{B}^{\mathrm{i}}(\mathrm{v}) \dot{\mathrm{v}} \\
& \dot{\mathbf{u}}^{\mathrm{i}}=\mathbf{C}^{\mathrm{i}}(\mathrm{v}) \mathbf{w}+\mathbf{D}^{\mathrm{i}}(\mathrm{v}) \dot{v}
\end{aligned}
$$

where

$$
\begin{aligned}
& \mathbf{A}^{\mathrm{i}}(\mathrm{v})=\mathbf{A}(\mathrm{v})-\mathbf{B}(\mathrm{v}) \mathbf{D}^{-1}(\mathrm{v}) \mathbf{C}(\mathrm{v}), \mathbf{B}^{\mathrm{i}}(\mathrm{v})=-\mathbf{B}(\mathrm{v}) \mathbf{D}^{-1}(\mathrm{v}) \\
& \mathbf{C}^{\mathrm{i}}(\mathrm{v})=\mathbf{D}^{-1}(\mathrm{v}) \mathbf{C}(\mathrm{v}), \quad \mathbf{D}^{\mathrm{i}}(\mathrm{v})=\mathbf{D}^{-1}(\mathrm{v})
\end{aligned}
$$

with $\mathbf{C}(\mathrm{v})=\left[\begin{array}{ll}\varepsilon_{\mathrm{c}} & 1\end{array}\right], \mathbf{D}(\mathrm{v})=\varepsilon_{\mathrm{d}}$. Note that the values of $\varepsilon_{\mathrm{d}}$ and $\varepsilon_{\mathrm{c}}$ used when deriving the approximate inverse are design parameters which determine the accuracy of the inversion achieved. Analogously to linear pole-zero inversion of systems with relative degree greater than zero, the approximate inverse contains high frequency poles to ensure that it is realisable. Roughly speaking, $\varepsilon_{\mathrm{d}}$ determines the frequency of the poles and $\varepsilon_{\mathrm{c}}$ influences the damping. Since the direct coupling term is zero in (3) (the relative degree is greater than zero), the inversion error tends to zero as $\varepsilon_{\mathrm{d}}$ and $\varepsilon_{\mathrm{c}}$ tend to zero. In the present example, values of 5 and 0.2 , respectively are found to achieve an adequate degree of accuracy.

Design of $C$ The approximate inverse, $\mathrm{G}^{-1}$, is augmented with controller dynamics, $\mathrm{C}$, consisting simply of integral action. The gain, $\mathrm{K}$, of the integrator is selected such that the inner loop bandwidth is around $130 \mathrm{rad} / \mathrm{s}$, see figure 2 .

Controller Realisation The velocity-based inverse, (5), cannot be directly implemented owing to the derivative action at the input (shown in figure 3a). However, owing to the integral action in the controller dynamics, $\mathrm{C}$, the controller structure in figure $3 \mathrm{a}$ may be equivalently formulated as in figure $3 b$ and is now realisable. Note that a direct link is maintained between the gains in the controller implementation and the gains in the original (unrealisable) design.

\section{Outer-loop}

The lateral acceleration is related to lateral velocity and fin angle by 


$$
\dot{\eta}_{\mathrm{y}}=\left(\mathrm{a}_{1}+2 \mathrm{a}_{2}|\mathrm{v}|+\mathrm{a}_{4} \mathrm{u} \operatorname{sgn}(\mathrm{v})\right) \mathrm{w}_{\mathrm{v}}+\left(\mathrm{a}_{4}|\mathrm{v}|+\mathrm{a}_{5}\right) \dot{\mathrm{u}}(6)
$$

Owing to the relatively high bandwidth of the inner loop, the lateral velocity can be considered effectively equal to the lateral velocity demand input to the inner loop controller. An outer loop controller with the simple structure shown in figure 4 is therefore sufficient. The nonlinear gain, $\mathrm{g}_{\mathrm{n}}$ includes a factor $\frac{1}{\left(a_{1}+2 a_{2}|v|\right)}$ to compensate for the nonlinear gain relating acceleration to velocity in (6). An additional factor is included to compensate for the effect of the direct coupling term in (6) at low frequencies, namely

$$
\hat{\mathrm{g}}_{\mathrm{nl}}=\sum_{\mathrm{i}=1}^{4} \mathrm{w}_{\mathrm{i}}(|\mathrm{v}|) \mathrm{g}_{\mathrm{i}}
$$

with $\mathrm{g}_{1}=1, \mathrm{~g}_{2}=2, \mathrm{~g}_{3}=5, \mathrm{~g}_{4}=5$ and triangular weighting functions, $\mathrm{w}_{\mathrm{i}}$ centred on lateral velocities of $1 \mathrm{~m} / \mathrm{s}$, $30 \mathrm{~m} / \mathrm{s}, 50 \mathrm{~m} / \mathrm{s}$ and $60 \mathrm{~m} / \mathrm{s}$. The overall nonlinear control gain, $g_{n l}$, is therefore $\frac{g_{n l}}{\left(a_{1}+2 a_{2}|v|\right)}$. The nonlinear gain is augmented with controller dynamics consisting of integral action. The gain of the integrator is selected such that the outer loop bandwidth is around $20 \mathrm{rad} / \mathrm{s}$, see figure 5 .

Controller Realisation The outer loop controller also includes integral action and so can be realised directly as discussed previously.

\section{PERFORMANCE}

Typical step responses of the controlled system are shown in Figure 6. It can be seen that the performance requirements are satisfied over the operating envelope $( \pm 50 \mathrm{~g})$. Typical stability margins and open-loop crossover frequencies of the velocity-based linearisations of the inner and outer control loops are

\begin{tabular}{|c|c|c|c|}
\hline \multicolumn{4}{|c|}{ Inner loop } \\
\hline $\begin{array}{c}\text { lateral } \\
\text { velocity } \\
(\mathrm{m} / \mathrm{s})\end{array}$ & $\begin{array}{c}\text { gain } \\
\text { margin } \\
(\mathrm{dB})\end{array}$ & $\begin{array}{c}\text { phase } \\
\text { margin } \\
(\mathrm{deg})\end{array}$ & $\begin{array}{c}\text { cross-over } \\
\text { frequency } \\
(\mathrm{rad} / \mathrm{s})\end{array}$ \\
\hline 0 & 9.35 & 72.7 & 130.8 \\
\hline 20 & 9.23 & 72.9 & 128.6 \\
\hline 50 & 9.60 & 73.5 & 123.9 \\
\hline
\end{tabular}

\begin{tabular}{|c|c|c|c|}
\hline \multicolumn{4}{|c|}{ Outer loop } \\
\hline $\begin{array}{c}\text { lateral } \\
\text { velocity } \\
(\mathrm{m} / \mathrm{s})\end{array}$ & $\begin{array}{c}\text { gain } \\
\text { margin } \\
(\mathrm{dB})\end{array}$ & $\begin{array}{c}\text { phase } \\
\text { margin } \\
(\mathrm{deg})\end{array}$ & $\begin{array}{c}\text { cross-over } \\
\text { frequency } \\
(\mathrm{rad} / \mathrm{s})\end{array}$ \\
\hline 0 & 12.3 & 57.3 & 17.3 \\
\hline 20 & 14.80 & 55.6 & 18.7 \\
\hline 50 & 14.28 & 55.3 & 18.7 \\
\hline
\end{tabular}

The stability margins vary due to the approximate nature of the particular inverse dynamics used and owing to the inaccurate inversion that results from using a scheduled approximation to the exact plant dynamics.

\section{SUMMARY}

The application of velocity-based gain-scheduling techniques to a demanding, highly nonlinear, missile control design task is investigated. Scheduling on instantaneous incidence (a rapidly varying quantity) is well known to lead to considerable difficulties with conventional gain-scheduling methods. Moreover, the missile dynamics do not have well-defined relative degree at operating points where the lateral velocity is zero and thus conventional feedback linearisation methods cannot be applied to design a controller. It is demonstrated that the velocity-based framework can, however, be used to successfully design a gainscheduled controller which achieves the performance requirements while maintaining the continuity with linear methods which is the principle advantage of classical gain-scheduling approaches.

\section{ACKNOWLEDGEMENT}

D.J. Leith acknowledges the generous support provided by the Royal Society for the work presented.

\section{REFERENCES}

HUNT, K.J.,JOHANSEN,T.A., 1997, Design and analysis of gain-scheduled control using local controller networks. International Journal of Control, 66, 619-651.

JOHANSEN, T.A., MURRAY-SMITH,R., 1997, The operating regime approach to nonlinear modelling and Control. Multiple Model Approaches to Modelling \& Control (Ed. Murray-Smith, R., Johansen, T.A.) (Taylor \& Francis, London).

LEITH, D.J., LEITHEAD, W.E., 1998a, Gain-Scheduled \& Nonlinear Systems: Dynamic Analysis by Velocity-Based Linearisation Families. International Journal of Control, 70, 289-317. 1998b, Gain-Scheduled Controller Design: An Analytic Framework Directly Incorporating Non-Equilibrium Plant Dynamics. International Journal of Control, 70, 249269.1999a, Analytic Framework for Blended Multiple Model Systems Using Linear Local Models. International Journal of Control, 72, 605-619. 1999b, Input-Output Linearisation by Velocity-Based Gain-Scheduling. International Journal of Control, 72, 229-246.

MCLEAN, D., 1990, Automatic Flight Control Systems. Prentice-Hall, London, 1990.

Tsourdos, A., Blumel,A. White, B.A., 1998, Trajectory Control of a Non-linear Homing Missile. Proceeding of the 14th IFAC Symposium on Automatic Control in Aerospace,pp.118-123, Aug. 1998, Korea.

White, B.A., Tsourdos, A., BlumelA., 1998, Lateral

Acceleration Control Design of a Non-linear Homing Missile. Proceeding of the 4th IFAC Nonlinear Control Systems Design Symposium, pp.707-712, July 1998, The Netherlands. 


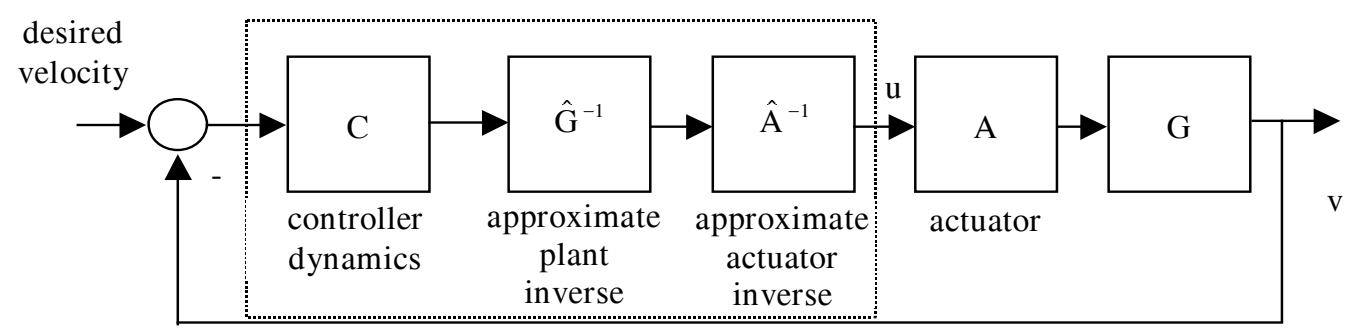

Figure 1 Structure of inner-loop controller
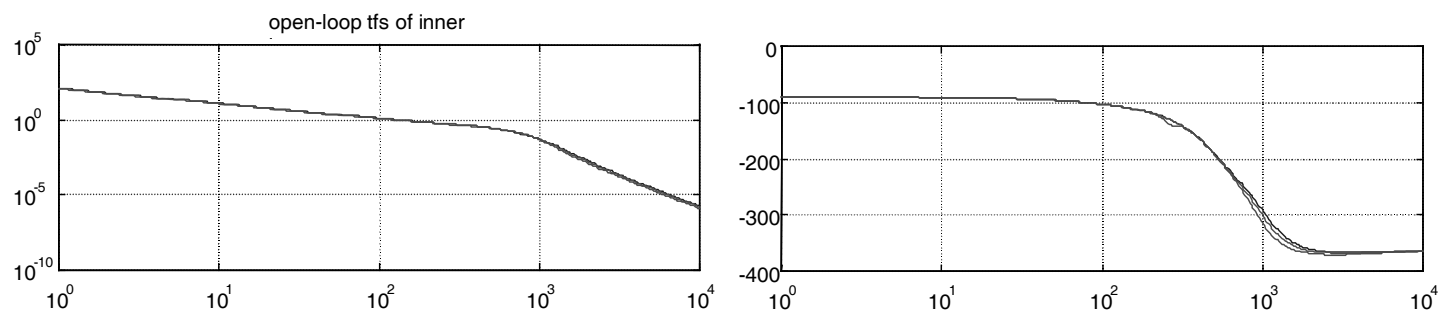

Figure 2 Transfer functions of open-loop velocity-based linearisations of inner-loop at lateral velocities of $0 \mathrm{~m} / \mathrm{s}, 20$ $\mathrm{m} / \mathrm{s}$ and $50 \mathrm{~m} / \mathrm{s}$ (corresponding roughly to lateral accelerations of $0 \mathrm{~g}, 20 \mathrm{~g}$ and $50 \mathrm{~g}$ ). The uniform nature of the dynamics is clearly evident.

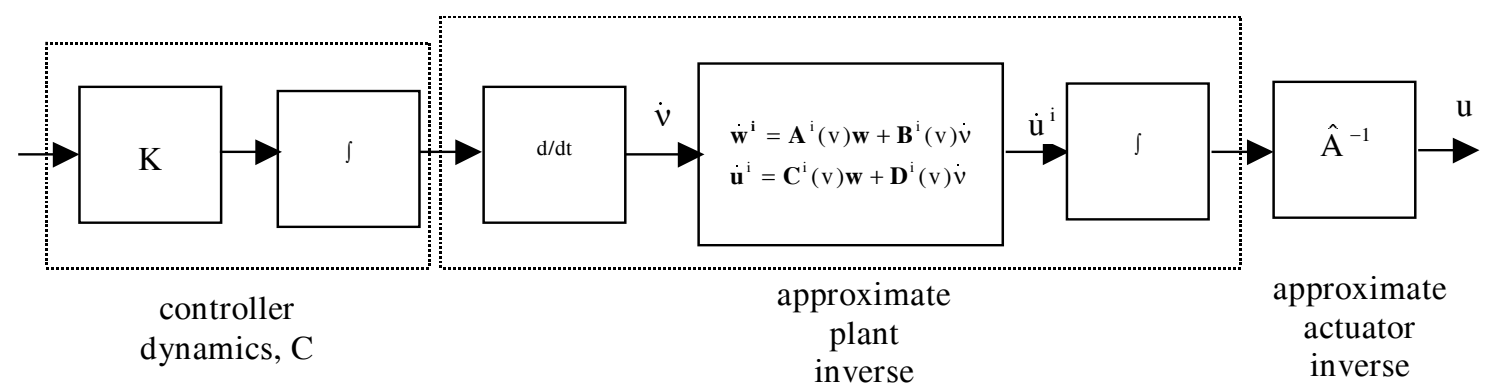

(a)

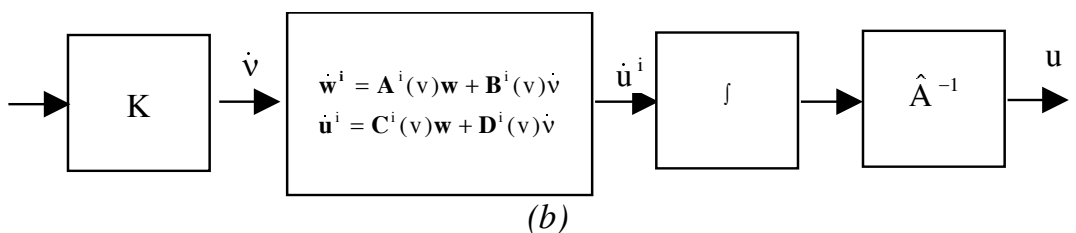

Figure 3 Alternative formulations of controller

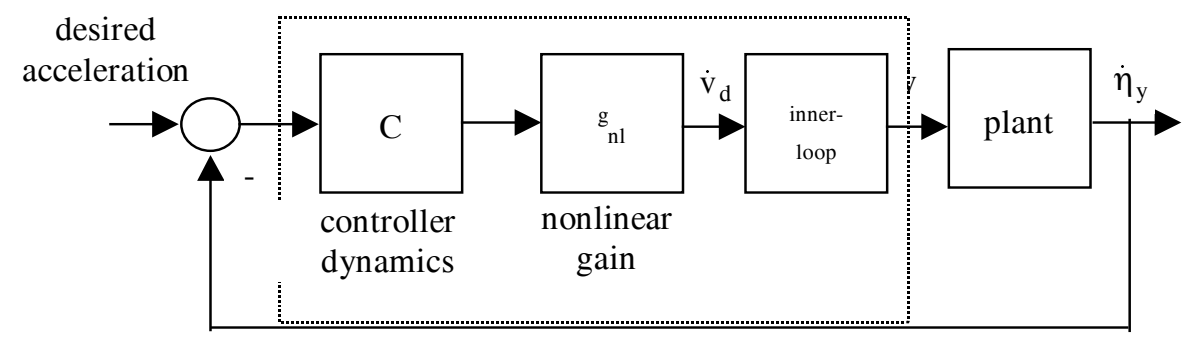

Figure 4 Structure of outer loop controller 

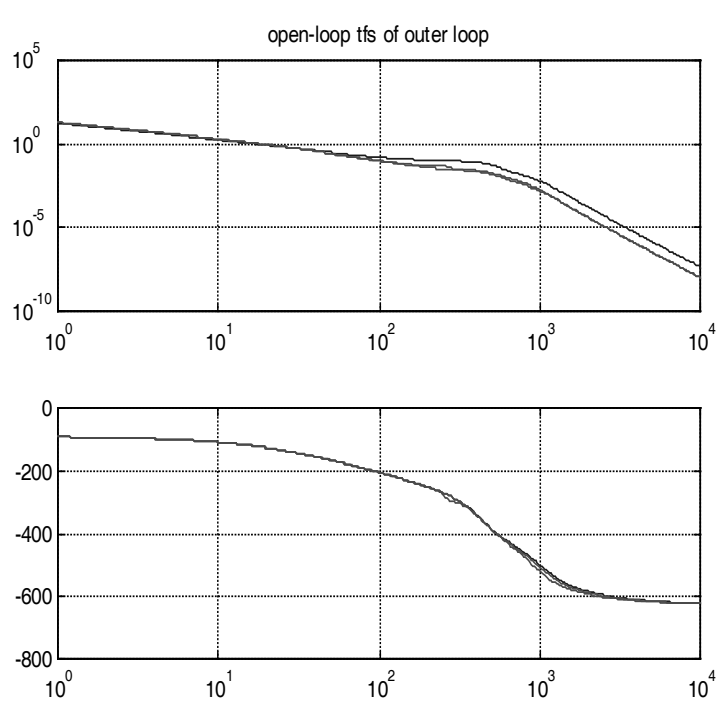

Figure 5 Transfer functions of open-loop velocitybased linearisations of outer-loop at lateral velocities of $0 \mathrm{~m} / \mathrm{s}, 20 \mathrm{~m} / \mathrm{s}$ and $50 \mathrm{~m} / \mathrm{s}$ (corresponding roughly to $0 \mathrm{~g}, 20 \mathrm{~g}$ and $50 \mathrm{~g}$ ). It can be seen that the dynamics are relatively uniform at frequencies below around 60 $\mathrm{rad} / \mathrm{s}$; that is, at frequencies up to and above the crossover frequency of the outer-loop.

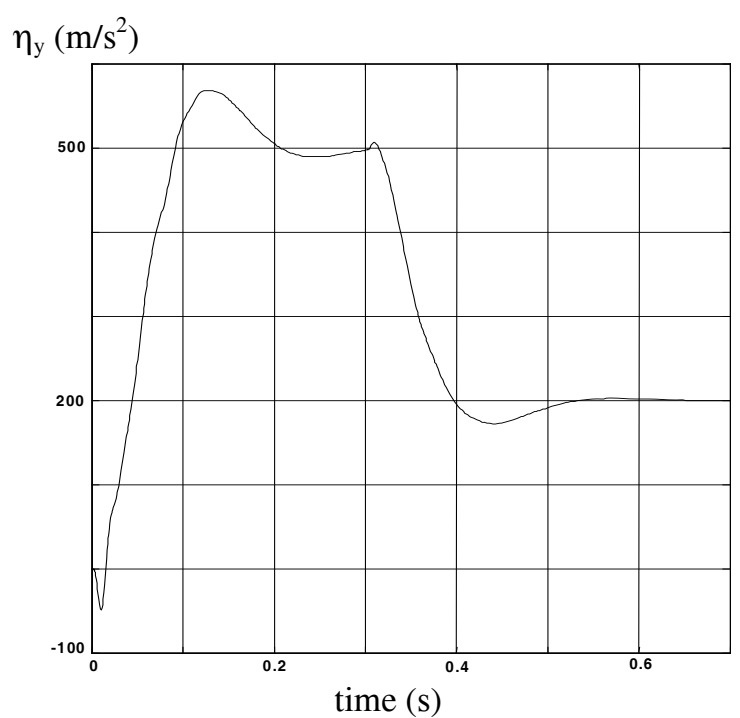

Figure 6 Step responses with velocity-based gainscheduled controller 